\title{
Second-order geometric continuous processing of singular points
}

\author{
Meng Biao ${ }^{1, \mathrm{a}}$, Liu $\mathrm{Hao}^{2, \mathrm{~b}}$, Tang Yuehong ${ }^{3, \mathrm{c}^{*}}$ and Gu Yuping ${ }^{4, \mathrm{c}}$ \\ ${ }^{1,3,4}$ College of Science, Nanjing University of Aeronautics and Astronautics, China
}

${ }^{2}$ College of Mechanical and Engineering, Nanjing University of Aeronautics and Astronautics, China

$\mathrm{c}^{\star}$ email: tangyuehong@nuaa.edu.cn (Corresponding author)

Keywords: subdivision surfaces, geometric continuity, singular points, algorithm

Abstract. This paper presents a shape adjustable C-C subdivision algorithm by introducing adjusting parameter $c(0 \leq c \leq 1)$. Based on this algorithm, this paper proposes a shape adjustable $G^{2}$ surface modeling method with explicit solutions of Bézier control points by taking 2-ring singular points as the control grid and using cyclic mapping. Compared with the existing, this new method has more advantages, which can not only obtain surfaces reaching $G^{2}$, but also solve the problem of adjustable surface design at the singular points.

\section{Introduction}

The singular point is unavoidable in surface modeling techniques. Due to its presence, the resulting surfaces could become "fold" seriously affecting the quality of the surfaces and causing industrial products useless. Therefore, the processing technology of singular points is very crucial in surface modeling. Because the traditional subdivision method for singular points only meets $G^{1}$ continuous, a lot of efforts have been made to construct $G^{2}$ continuous surfaces.In 2009, Thomas J. Cashman of the University of Cambridge used any number of degree B-spline curve to advance a symmetrical non-uniform subdivision algorithm. What is more, by using the same algorithm in surfaces, they got an odd-order symmetric non-uniform subdivision algorithm. However this algorithm had no specific explains about singular points, and was only useful in odd-order situation. Then Thomas J.Cashman and Malcolm A.Sabin ${ }^{[1]}$ designed a new subdivision format to handle singular points of non-uniform subdivision algorithm, which could not get ideal results and had poor locality. Subsequently, Liu hao ${ }^{[2]}$ used manifold method and subdivision method to construct a $G^{2}$ continuous free-type surface, achieving the integration of manifold surfaces and Catmull-Clark subdivision surfaces, but the location information of singular points had not been considered when constructing $\mathrm{n}$ - sided domain patch. Based on the Charles Loop 's study ${ }^{[3]}$, this paper makes the following improvements: (i) This paper introduces a shape adjustable Catmull-Clark subdivision method to make surfaces "hybrid subdivision", so that the final fitting surface would be adjustable; (ii) The cyclic matrix contains parameter $\mathrm{c}$ when we use circular mapping to deal with singular points around the surface. As a result, each surface piece is adjustable; (iii)This paper provides the location of double seventh degree Bézier control points and the explicit solution of circulation equations. Furthermore, the explicit representation of each surface piece is put forward and the singular point $G^{2}$ filling effect is given.

\section{An shape adjustable C-C subdivision surface}

In order to enhance the surface design flexibility and adjustability, this section will extend curve adjustable ${ }^{[4]}$ to the surface of the case and re-design geometric and topology rules .

For any topological shape of a control grid, the new subdivision rules still consists of two parts: the production of geometry points and the establishment of topological structure.

\section{Generation of geometric points}

Given an initial mesh $\Gamma^{(0)}$, We obtained control mesh as $\Gamma^{(k)}$ after improvement $k$ times Catmull-Clark subdivision. We consider $\Gamma^{(k)}$ as the degree is $n$ and vertices are $v^{(k)}$ of the local grid. Its neighborhood is by $n$ edge-vertex $e_{1}^{(k)}, \mathrm{L}, e_{n}^{(k)}$, and ${ }_{n}$ coplanar vertices $f_{1}^{(k)}, \mathrm{L}, f_{n}^{(k)}$ composition. Then, after the $k+1$ times subdivision produced the new pasta point (F- points): 
$f_{i}^{(k+1)}=\frac{1}{4}\left(v^{(k)}+f_{i}^{(k)}+e_{i}^{(k)}+e_{i+1}^{(k)}\right)$, the new edge point (E- points): $e_{i}^{(k+1)}=\frac{v^{(k)}+e_{i}^{(k)}}{2} c+\frac{f_{i-1}^{(k+1)}+f_{i+1}^{(k+1)}}{2}(1-c)$

and the new vertex (F- points): $v^{(k+1)}=\frac{4 c Q}{n}+\frac{8 c(1-c) R}{n}+\frac{n-4 c(2-c) v^{(k)}}{n}$, where $Q$ is the average of all the old vertices to the adjacent surface new pasta point, $R$ represents the average of all post on the old side of the midpoint of the vertices, $n$ is corresponding vertices degree. At the same time $i=1, \mathrm{~L}, n$, and set $e_{n+1}^{(k)}=e_{1}^{(k)}, f_{n+1}^{(k)}=f_{1}^{(k)}$.

\section{Establishment of topological structure}

From the sub-surface of the rules can be introduced, given the original control point $d_{i j}(i, j=0, \mathrm{~L}, n)$, then ,after the subdivision surface can be expressed as

$$
P(u, v)=U C G C^{T} V^{T},
$$

where, $U=\left[\begin{array}{llll}1 & u & u^{2} & u^{3}\end{array}\right], V=\left[\begin{array}{llll}1 & v & v^{2} & v^{3}\end{array}\right]$, the corresponding parameter adjustment matrix as

$$
C=\frac{1}{2(1+c)}\left[\begin{array}{cccc}
c & 2 & c & 0 \\
-2 c(2-c) & 0 & 2 c(2-c) & 0 \\
c(5-4 c) & (-2 c+3)(c-2) & 4 c^{2}-11 c+6 & c(2 c-1) \\
2 c(c-1) & 2(c-1)(c-2) & -2(c-1)(c-2) & 2 c(1-c)
\end{array}\right] .
$$

\section{$G^{2}$ processing of singular points in surface subdivision}

This section improves Charles Loop theory and gives a $G^{2}$ surface reconstruction method with singular points. First, the initial control mesh "hybrid subdivision", that is, using shape adjustable $\mathrm{C}-\mathrm{C}$ subdivision method for surface and then using the classic $\mathrm{C}-\mathrm{C}$ subdivision, the purpose is to ensure surface patch having B-spline boundaries; Secondly, using the method of cyclic mapping we obtain constraint equations of $G^{2}$ continuity with 2-ring of singular point as a control grid, ;Finally, by energy function optimization method we give a explicit solution of Bézier control points for a $G^{2}$ filling $n$ side hole.It is not only $G^{2}$ around a singular point but also $G^{2}$ connection between the patch and the Catmull-Clark subdivision surfaces, and whole surface becomes $G^{2}$ continuous.

\section{Second-order geometric continuity}

According to the geometric continous definition ${ }^{[5]}$, The necessary and sufficient condition for two patches $P_{i}(u, v)$ and $P_{i+1}(u, v):[0,1] \times[0,1] \rightarrow R^{m}$ be $G^{2}$ along the common boundary $C: C(v)=P_{i}(1, v)=P_{i+1}(0, v)$ is that existing $\left\{\left(p_{i}(v), q_{i}(v)\right), i=1,2\right\}$ such that

$$
\frac{\partial^{2}}{\partial u^{2}} P_{i}=p_{2}(v) \frac{\partial}{\partial u} P_{i+1}+q_{2}(v) \frac{\partial}{\partial v} P_{i+1}+p_{1}^{2}(v) \frac{\partial^{2}}{\partial u^{2}} P_{i+1}+2 p_{1}(v) q_{1}(v) \frac{\partial^{2}}{\partial u \partial v} P_{i+1}+q_{1}^{2}(v) \frac{\partial^{2}}{\partial v^{2}} P_{i+1} \text {, along } C .
$$

\section{"Hybrid subdivision" of the initial control mesh}

We can find control grid for any topological shapes scattered points using C-C subdivision algorithm given in Section 1 , from this, the mesh surface can be expressed as

$$
P(u, v)=U C H \mathrm{C}^{T} V^{T}
$$

Set $A=C H C^{T}, H$ is bicubic coefficient matrix and the $k-t h$ patch corresponding coefficient matrix is $A_{k}=C_{k} H_{k} C_{k}^{T}$,,where $C_{k}$ is the resulting matrix by substituting $c_{k}$ for $c$ in equ. (2).

Subdivision again and this process is known as "hybrid subdivision". By "hybrid subdivision" isolating singular point from the regular grid, constructing a n-sided hole of singular point. For a regular place already satisfy the second order continuous, next thing is how to ensure a second order geometric continuous case filling with n-sided holes and after connect with patch around the whole surface to achieve second-order continuous. As shown in Fig. 1. 


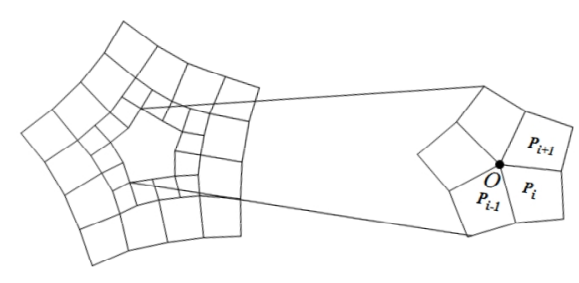

Fig. 1 getting n-side hole from "hybrid subdivision"

In Fig. 1, connected directly to the singular point called the edge of the internal boundary; and connected indirectly to the singular point called the external boundary.Finally, to make the whole surface $G^{2}$ continuous, requiring patch boundary between the internal connection is $G^{2}$ and the external boundary connected with Catmull-Clark between patches also satisfies $G^{2}$.For filling "n-side hole",this paper constructs a double 7-degree B- spline surface patches to fill, thus

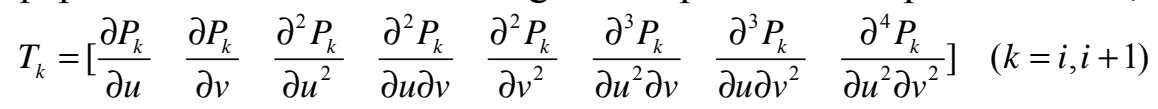

Let $\Theta_{i}$ be the parametric matrices of patches $P_{i}$ and $P_{i+1}$, by $G^{2}$ definition in section 2.1 , we have $T_{i}=T_{i+1} \cdot \Theta_{i}$, thus there exist a common vertex of the $n$ patches $p_{i}(i=0, \mathrm{~L}, n-1)$ with the geometric continuity and cycling conditions. Then $T_{0}=T_{0} \cdot \Theta_{n-1} \cdot \Theta_{n-2} \cdot \mathrm{L} \cdot \Theta_{1} \cdot \Theta_{0}$ on $n$ common vertexes between the patches holds. From the type, $I=\Theta_{n-1} \cdot \Theta_{n-2} \cdot \mathrm{L} \cdot \Theta_{1} \cdot \Theta_{0}$.

To make the whole surface $G^{2}$, requiring patch boundary between the internal connection is $G^{2}$ and the external boundary connected with Catmull-Clark also satisfies $G^{2}$.

\section{Solving Bézier control points}

Let $C$ be coefficient matrix of control points for double seventh degeree patch, then $p_{i}$ is a coefficient matrix of the corresponding $i$-th patch. When $C P=W A$, the overall patch is $G^{2}$, where $C$ is cyclic matrix , $P$ is control points of double seventh degree patch and $A$ is a spline vertexes of $i$-th patches in the 2- ring. For cyclic matrix ${ }^{[6]} C=\operatorname{bcirc}\left(c_{0}, c_{1}, \mathrm{~L}, c_{n-1}\right)$, all its characteristic roots are $\lambda_{i}=\sum_{j=0}^{n} c_{j} \omega_{i}^{j}$, where, $\omega_{j}=e^{i \frac{2 \pi}{n+1} j}$, and $C$ is similar to a diagonal matrix $\operatorname{diag}\left(\lambda_{0}, \mathrm{~L}, \lambda_{\mathrm{n}-1}\right)$.

In order to make the minimize of the quadratic energy function ${ }^{[7]}$, then use Lagrange multiplier method $^{[8]}$, the constraints can be transformed into the following form.

$$
\left\{\begin{array}{l}
{\left[\begin{array}{cc}
E & c_{0}^{T} \\
c_{0} & 0
\end{array}\right]\left[\begin{array}{l}
p_{0} \\
\lambda_{0}
\end{array}\right]+\left[\begin{array}{cc}
0 & 0 \\
c_{1} & 0
\end{array}\right]\left[\begin{array}{c}
p_{1} \\
\lambda_{1}
\end{array}\right]+\mathrm{L}+\left[\begin{array}{cc}
0 & c_{1}^{T} \\
0 & 0
\end{array}\right]\left[\begin{array}{c}
p_{n-1} \\
\lambda_{n-1}
\end{array}\right]=\left[\begin{array}{c}
0 \\
\omega_{0}
\end{array}\right] a_{0}+\left[\begin{array}{c}
0 \\
\omega_{1}
\end{array}\right] a_{1}+\mathrm{L}+\left[\begin{array}{c}
0 \\
\omega_{n-1}
\end{array}\right] a_{n-1}} \\
{\left[\begin{array}{cc}
0 & c_{1}^{T} \\
0 & 0
\end{array}\right]\left[\begin{array}{c}
p_{0} \\
\lambda_{0}
\end{array}\right]+\left[\begin{array}{cc}
E & c_{0}^{T} \\
c_{0} & 0
\end{array}\right]\left[\begin{array}{c}
p_{1} \\
\lambda_{1}
\end{array}\right]+\mathrm{L}+\left[\begin{array}{cc}
0 & 0 \\
0 & 0
\end{array}\right]\left[\begin{array}{c}
p_{n-1} \\
\lambda_{n-1}
\end{array}\right]=\left[\begin{array}{c}
0 \\
\omega_{n-1}
\end{array}\right] a_{0}+\left[\begin{array}{c}
0 \\
\omega_{0}
\end{array}\right] a_{1}+\mathrm{L}+\left[\begin{array}{c}
0 \\
\omega_{n-2}
\end{array}\right] a_{n-1}} \\
{\left[\begin{array}{ll}
0 & 0 \\
c_{1} & 0
\end{array}\right]\left[\begin{array}{l}
p_{0} \\
\lambda_{0}
\end{array}\right]+\left[\begin{array}{cc}
0 & 0 \\
0 & 0
\end{array}\right]\left[\begin{array}{c}
p_{1} \\
\lambda_{1}
\end{array}\right]+\mathrm{L}+\left[\begin{array}{cc}
0 & c_{1}^{T} \\
0 & 0
\end{array}\right]\left[\begin{array}{l}
p_{n-2} \\
\lambda_{n-2}
\end{array}\right]+\left[\begin{array}{cc}
E & c_{0}^{T} \\
c_{0} & 0
\end{array}\right]\left[\begin{array}{c}
p_{n-1} \\
\lambda_{n-1}
\end{array}\right]=\left[\begin{array}{c}
0 \\
\omega_{1}
\end{array}\right] a_{0}+\left[\begin{array}{c}
0 \\
\omega_{2}
\end{array}\right] a_{1}+\mathrm{L}+\left[\begin{array}{c}
0 \\
\omega_{0}
\end{array}\right] a_{n-1}}
\end{array}\right.
$$

For the i-th block equations $, \sum_{i=0}^{n-1} c_{i}^{T} \lambda_{i}+\sum_{i=0}^{n-1} E p_{i}=0, \sum_{i=0}^{n-1} c_{i} p_{i}=\sum_{i=0}^{n-1} \omega_{i} a_{i}$, where,for the i-th equation, only coefficient of the $p_{i}$ is $E$, the rest are zero. Then the corresponding trans double seventh degree control points $p_{i}$ are obtained by DFT and IDFT ${ }^{[9]}$. 


\section{Surface Modeling Results}

In the section, surface modeling results for a car part with 1452 local scattered points is present by using the above method. There are 323 quadrilateral meshes and polygon filling of the 10 singular points after "hybrid subdivision" . Fig. 2 (a) is quadrilateral control mesh after "hybrid subdivision" , Fig. 2 (b) is two-ring structure of the singular point, and Fig. 2 (c) is the patch around the singular point meet $G^{2}$ after double seventh degree spline fitting. The process is as shown in Fig. 2.

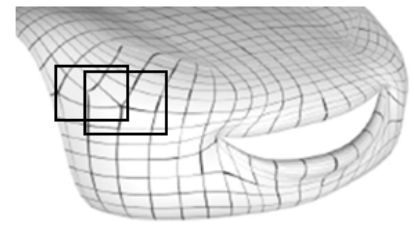

(a)"hybrid subdivision"

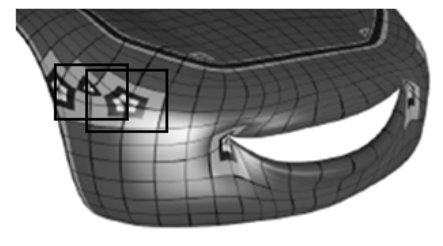

(b) filling of n-sided hole



(c) $G^{2}$ continuous surface

Fig. $2 G^{2}$ continuous surface modeling results

\section{Summary}

This paper focuses on discusses about second order gemetric continuous processing of singular points in subdivision surface modeling, and puts forward a $G^{2}$ surface reconstruction method with singular points in subdivision. It includes the following parts: first of all, it advances the shape adjustable C-C subdivision algorithm; secondly, it proposes the $G^{2}$ reconstruction method of singular points; finally, it obtains the results of the filling of 3-sided hole and 5-side hole. It has the adjustable properties for subdivision surfaces in industrial design and extends the application fields.

\section{Acknowledgement}

This work was supported by the National Nature Science Foundation of China under Grant No. 51175248/E050603.

\section{References}

[1] T.J. Cashman, N.A. Dodgson, M.A. Sabin, Selective knot insertion for symmetric , non-uniform , refine and smooth B-splines subdivision ,Computer Aided Geometric Design,26(1) (2009), 472-472 . [2] Liu Hao, LiaoWenhe, Constructing a continuous and free surface by C-C subdivision method and manifold method, Journal of Computer Aided Design \& Computer Graphics, 17 (4)( 2005),644-649. [3] Charles Loop. Second order smoothness over extraordinary vertices, Proceedings of the 2004 Eurographics/ACM Siggraph Symposium on Geometry Processing, 2004,1(71), pp.165- 174.

[4] Wang Weimin ,Luo Guoming ,Zhang Jiwen ,An adjustable Catmull-Clark subdivision surfaces, Journal of ENGINEERING GRAPHICS, 3(2002),115-121.

[5] Wang Guojin, Wang Guozhao ,Zheng Jianmin .Computer Aided Geometric Design , Higher Education Press, Beijing;Springer, Heidelberg,2001.

[6] Lu Chengbo, Research on fast algorithms of several circulant matrices, $\mathrm{PhD}$ thesis ,Shanghai University, 2012.

[7] M .Eck, H .Hoop, Automatic reconstruction of B-spline surfaces of arbitrary topological type, J.Computer Graphics, 30(4)(1996), 325-334.

[8] Yong Junhai ,Cheng Fuhua (Frank).Adaptive subdivision of Catmull-Clark subdivision surfaces, Computer-Aided Design \& Applications, 2005,2(1-4), 253-261.

[9] Li Wenxin, David Zhang, Xu Zhuoqun, Palmprint recognition based on fourier transform, Journal of Software, 13 (5)( 2002), 879-886. 